Volume 2

Issue 4 -- Integrative Medicine

Article 34

$11-20-2015$

\title{
Fair Weight Loss After Gastric Rebanding for Slippage
}

\author{
Ahmed Dalmar \\ Maharaj Singh \\ Sara K. Roloff \\ Thomas Y. Chua
}

Follow this and additional works at: https://aah.org/jpcrr

Part of the Medical Anatomy Commons, Medical Pathology Commons, and the Other Medicine and Health Sciences Commons

\section{Recommended Citation}

Dalmar A, Singh M, Roloff SK, Chua TY. Fair Weight Loss After Gastric Rebanding for Slippage. J Patient Cent Res Rev 2015;2:214-215. http://dx.doi.org/10.17294/2330-0698.1244

Published quarterly by Midwest-based health system Advocate Aurora Health and indexed in PubMed Central, the Journal of Patient-Centered Research and Reviews (JPCRR) is an open access, peer-reviewed medical journal focused on disseminating scholarly works devoted to improving patient-centered care practices, health outcomes, and the patient experience. 
ACM for two targeted family medicine residency teaching clinics.

Results: Through February 2015, 29 providers systemwide completed the module with 212 in progress. Data from targeted clinics demonstrated system level increases in all metrics over project period, with average increases of $21 \%$ in ACT completion, $34 \%$ in use of AAP and 7\% in ACM use. Participants' evaluations are strong: $80 \%$ of module completers rate MOC training as yielding a high return on their time investment; $100 \%$ of M3 students report completing an ACT test and an associated impact on their patient's care. Participant comments include: “... since completing this project I will strive to screen all my asthma patients at every visit ... [and] adjust their medications based on it. [It's] an extremely useful clinical tool"; and "I plan on trying to use [the] ACT with more appointments as a way to check up on asthma quickly. Score increased to $44 \%$ with minimal intervention."

Conclusion: Aligning physician education opportunities across the continuum with health care system metrics meets board (re)certification requirements, residency and medical student accreditation requirements and improves care for patients.

\section{Incidence of Breast, Colorectal and Lung Cancers and Mortality Among Women Within Midwestern States}

Ruth M. Perez, Matthew Rappelt, Kathryn Kossow, Maharaj Singh

\section{Aurora Research Institute, Aurora Health Care}

Background: Breast, colorectal and lung cancers have been shown to be the most common cancers as well as the leading causes of cancer death among women. Previous studies suggest that the Northeast had significantly higher rates in incidence and mortality than the Midwest, South and Western regions. However, new data indicates that the Midwest now harbors the highest mortality rates. In Wisconsin, the sixth largest state in the Midwest, cancer is the leading cause of death. Differences in incidence and mortality of breast, colorectal and lung cancers have been observed between Wisconsin, other Midwestern states and national data, warranting further investigation.

Purpose: To examine the incidence and mortality of Wisconsin females across breast, colorectal and lung cancers compared to that of the national average along with the individual states that comprise the Midwest (ND, SD, $\mathrm{NE}, \mathrm{KS}, \mathrm{MN}$, IA, MO, IL, IN, OH and MI).

Methods: Female incidence and mortality rates were retrieved from the Centers for Disease Control and Prevention (CDC) National Program of Cancer registries for the 2011 year, while census data was retrieved from the U.S. Census Bureau for the nation, region and individual states. Data was analyzed using two-sample z-test for proportions with significance set at $\mathrm{P}<0.05$.

Results: Compared to the national incidence of breast cancer
(122 per 100,000), Wisconsin women had a significantly higher incidence $(\mathrm{P}<0.05)$. Within the Midwest, Wisconsin had a higher incidence than Indiana $(\mathrm{P}<0.0005)$ as well as higher mortality than Nebraska $(\mathrm{P}<0.05)$. However, Wisconsin had lower incidence of breast cancer than Minnesota and Ohio $(\mathrm{P}<0.01)$ and lower mortality than Ohio $(\mathrm{P}<0.05)$. Wisconsin had both lower incidence and mortality than Illinois, Indiana, Iowa and Nebraska $(\mathrm{P}<0.05)$ for colorectal cancer. For lung cancer, Wisconsin had a higher incidence and mortality than Minnesota and Nebraska $(\mathrm{P}<0.005)$ and lower incidence than Indiana, Michigan and Missouri $(\mathrm{P}<0.05)$. No significant differences were noted between Wisconsin and other Midwestern states.

Conclusion: Though variations exist between Wisconsin and other Midwestern states in incidence and mortality, there are no consistent trends between these states and the three most common cancers. As a whole, however, the Midwest had statistically higher incidence and mortality rates than the nation. Further investigations into the regional differences between Wisconsin, the Midwest, and other states with similar demographic composition will be explored.

\section{Fair Weight Loss After Gastric Rebanding for Slippage}

\section{Ahmed Dalmar, Maharaj Singh, Sara K. Roloff, Thomas Y. Chua}

\section{Aurora Research Institute, Aurora Health Care; Wisconsin Bariatrics}

Background: Laparoscopic adjustable gastric banding (LAGB) is one of the most common bariatric surgery procedures performed in the United States. LAGB results in safe and satisfactory weight loss, but it is often complicated with slippage, a complication requiring rebanding (reoperation). There is a paucity of studies and no uniform consensus regarding weight loss after rebanding.

Purpose: This study assessed the effect of rebanding for slippage after LAGB on weight loss up to five years.

Methods: This is a historical cohort study of 865 patients who underwent LAGB from 2001 to 2011. Rebanding was performed in $103(11.9 \%)$ patients. Primary outcome of interest was percent excess weight loss (\% EWL), which was categorized as fair $(>25-50 \%)$ and failure $(<25 \%$ EWL) after rebanding. Of the 103 patients diagnosed with slippage, 23 were excluded from further analysis because either the band was removed $(n=15)$, or they were rebanded twice due to recurrent slippage $(n=2)$ or lack of enough data $(n=6)$. Of the remaining 80,76 patients were matched with 76 controls without slippage using propensity matching. Paired t-test was used to compare weights (initial, at reoperation, and 1,2, 3, 4 and 5 years before and after rebanding). Chisquare test was used to compare EWL rate between groups. Multivariate logistic regression was performed to determine predictive probability for propensity matching of slippage.

Results: The majority of patients were female $(82.9 \%)$. Mean age was $44.32 \pm 11.3$ years, mean preoperative body 
mass index $48.62 \pm 8.0 \mathrm{~kg} / \mathrm{m}^{2}$ and mean preoperative excess weight $171.28 \pm 52.0 \mathrm{lbs}$. Median follow-up after LAGB was 63.63 months (range $0.0-162.4$ ) for a total of 4,859 patientyears. During follow-up, 103 patients $(11.9 \%)$ underwent reoperation for slippage at a median of 54.26 months (range 0.0-160.50) after LAGB. We found a significantly lower weight at rebanding, and at 1, 2, 3, 4 and 5 years after rebanding in patients with slippage compared to their initial weight, and their weight at 1, 2, 3, 4 and 5 years after LAGB. There was a significantly lower excess weight loss failure rate in patients with slippage compared to matching controls ( $40 \%$ vs. $60 \%, \mathrm{P}=0.0006)$ after first year. There were no differences in EWL rate between the two groups after first year. In multivariate analysis only female gender was significantly associated with slippage.

Conclusion: Failure rate of excess weight loss after rebanding for slippage was lower or similar to the failure rate after initial laparoscopic adjustable gastric banding.

\section{Feasibility of Atrial Delivery and Tracking of Stem Cells in a Porcine Model}

Nina Garlie, Timothy Hacker, Eric G. Schmuck, Jill Koch, Jayant Khitha, Amish Raval, Indrajit Choudhuri

\section{Regenerative Medicine Center, Aurora Research Institute, Aurora Health Care; Department of Medicine, University of Wisconsin School of Medicine and Public Health; Aurora Cardiovascular Services, Aurora Health Care}

Background: Many patients undergoing open heart surgery have sinus node dysfunction and atrial fibrillation, leading to adverse outcomes. Mesenchymal stem cells (MSC) delivered at the time of surgery may have a reparative effect on atrial tissue, thereby improving sinus node function and reducing or preventing atrial fibrillation. Stem cell delivery to the atrium is entirely unstudied. This is a significant gap in medical research, as atrial disease contributes significantly to health care costs.

Purpose: The purpose of this pilot study is to establish a technique to deliver MSC to the atria through an open-chest model, to assess the safety of this technique, and to evaluate the acute retention of the delivered cells.

Methods: All in vivo animal experimentation was approved by the University of Wisconsin Animal Care and Use Committee and took place in the Cardiovascular Physiology Core Facility at UW-Madison. MSC (3-5×106 in $50 \mu 1$ per site) were injected intramyocardially during an open-chest procedure in anesthetized pigs. To track the cells in vivo, MSC were labeled with $18 \mathrm{FDG}$ then visualized at 1 and 6 hours postinjection by PET/CT. Pigs were monitored for intraoperative arrhythmia, bleeding and hypotension.

Results: By gently repositioning the heart, both atria were accessible for the injections. The thickest part of each atrium was isolated and stabilized briefly for the injection using a hemostat. The injected cells were visible by PET/CT 1 and 6 hours postinjection. However, when the MSC were labeled with $10 \mathrm{mCi} 18 \mathrm{FDG}$, the signal was too high, causing a bloom around the areas of injection. So the dose was lowered to $5 \mathrm{mCi} 18 \mathrm{FDG}$, which resulted in a clear signal at 1 hour in both atria. At 6 hours, the right atrial injection was still easy to read, but the left injection was difficult to resolve from background signal. All injections resulted in cell leakage from the injection site and uptake of the signal into the lungs. However, pulmonary function as measured by $\mathrm{SpO}_{2}$ and $\mathrm{EtCO}_{2}$ was unchanged. Intraoperative arrhythmias detected during the injections were caused by manipulation of the heart. No additional arrhythmias were detected. No bleeding or hypotension was observed as a result of the injections.

Conclusion: This pilot study demonstrated that atrial delivery of MSC is feasible and safe in an open-chest porcine model and that MSC are retained for at least 6 hours postinjection. Subsequent studies will determine the ability of MSC to downregulate inflammation, decrease scarring and prevent sinus node dysfunction.

\section{Does the Expression of Ki-67, p16 and COX-2 at Initial Diagnosis of Breast Atypia or Usual Ductal Hyperplasia Predict a Second Clinically Significant Event?}

Judy A. Tjoe, Philippe Gascard, Jianxin Zhao, Gary F. Neitzel, Maharaj Singh, Brittany Last, James Marx, Thea Tlsty, Sanjay Kansra

Surgical Breast Oncology, Aurora Health Care; Department of Pathology, University of California, San Francisco; ACL Laboratories, Department of Pathology, Aurora Health Care; Aurora Research Institute, Aurora Health Care

Background: Women diagnosed with atypical ductal hyperplasia $(\mathrm{ADH})$ or atypical lobular hyperplasia (ALH) have a fivefold increased risk of developing breast cancer. Because ADH/ALH can be precursors or predictive markers of a subsequent clinically significant event (SCSE), i.e. atypia, in situ or invasive carcinoma, the clinical outcome for these patients ranges anywhere from remission to invasive malignancy. Currently we cannot predict which atypical breast lesion is likely to be associated with future cancer, resulting in aggressive management and, possibly, overtreatment. Kerlikowske et al. reported that a combination of three biomarkers (cell cycle regulator p16INK4a, proliferation antigen Ki-67 and stress response enzyme COX-2) predicted risk of progression for $\sim 50 \%$ of women diagnosed with ductal carcinoma in situ and treated by lumpectomy alone.

Purpose: To evaluate whether expression levels of p16, Ki-67 and COX-2 predict risk of development of a SCSE in patients initially diagnosed with breast atypia (ADH or ALH) or usual ductal hyperplasia (UDH).

Methods: Patients with an initial diagnosis of pure $\mathrm{ADH} / \mathrm{ALH}$ were identified by medical record review and the lesion confirmed by a single pathologist blinded 